\title{
Elastic Scattering of Low-Energy Electrons by Carbon Disulphide
}

\author{
M. H. F. Bettega, ${ }^{\dagger}$ A. P. P. Natalense, ${ }^{\S}$ M. A. P. Lima*, and L. G. Ferreira* \\ $\dagger$ Departamento de Física, Universidade Federal do Paraná, \\ Caixa Postal 19081, 81531-990, Curitiba, Paraná, Brazil \\ $\stackrel{+}{+}$ Present address: A. A. Noyes Laboratory of Chemical Physics, \\ California Institute of Technology, Pasadena, California, 91125 \\ $\S$ Present address: Department of Chemistry, Texas A 85 M University, \\ 77843-3255 College Station, Texas \\ *Instituto de Física "Gleb Wataghin", \\ Universidade Estadual de Campinas, \\ Caixa Postal 6165, 13083-970, Campinas, São Paulo, Brazil
}

Received 4 August, 1999

\begin{abstract}
We report results from an ab initio calculation of low-energy electron scattering by $\mathrm{CS}_{2}$ molecules using the Schwinger multichannel method with pseudopotentials. We calculated elastic integral, differential and momentum transfer cross sections in an energy range from $5 \mathrm{eV}$ up to $50 \mathrm{eV}$ and compared our results with available theoretical results and experimental data. Through the symmetry decomposition of our integral cross section and eigenphase sum analysis, we found cross section peaks that may be interpreted as broad shape resonances in the cases of the $\Sigma_{g}, \Pi_{g}, \Pi_{u}$, and $\Delta_{u}$ symmetries. Among these possible resonances, the $\Pi_{g}, \Pi_{u}$, and $\Delta_{u}$ are being reported for the first time.
\end{abstract}

\section{Introduction}

Studies on electron-collision with $\mathrm{CS}_{2}$ have received little attention in the past years. We can quote the theoretical work of Lynch et al. [1], the measurements of total cross section by Szmytkowski [2], the measurements of elastic cross sections by Sohn et al. [3] and the more recent works by Raj and Tomar [4] and Lee et al.[5]. Lynch et al. used the continuum multiplescattering model and studied elastic $\mathrm{e}^{-} \mathrm{CS}_{2}$ scattering from $0 \mathrm{eV}$ up to $100 \mathrm{eV}$. They were able to find some shape resonances, especially the low-energy $\Pi_{u}$ shaperesonance around $1.85 \mathrm{eV}$. The measurements of Sohn et al. covered the energy range from $0.3 \mathrm{eV}$ up to $5 \mathrm{eV}$. Szmytkowski measured total cross sections covering the energy range from $0.4 \mathrm{eV}$ up to $80 \mathrm{eV}$ and Raj and Tomar applied the independent atom model to calculate cross section for energies above $100 \mathrm{eV}$. Lee et al. used the Schwinger variational iterative method combined with the distorted wave method to study $\mathrm{e}^{-}-\mathrm{CS}_{2}$ scattering. They calculated elastic and total (elastic plus inelastic) cross sections for energies up to $100 \mathrm{eV}$ and also calculated differential cross sections at selected energies.

In this work we present results of our calculations on elastic $e^{-}-\mathrm{CS}_{2}$ collision, obtained with the Schwinger multichannel method with pseudopotentials (SMCPP) [6] at the fixed-nuclei static-exchange approximation. We have considered energies ranging from $5 \mathrm{eV}$ up to $50 \mathrm{eV}$, thus avoiding the very low-energy range, where polarization effects are known to be important in the description of the scattering process. The SMCPP method has been applied with success in the calculation of elastic and inelastic cross sections for electron scattering by several molecular systems [7]. The symmetry decomposition of the integral cross section according to the irreducible representations of the molecular point group $\left(\mathrm{D}_{\infty h}\right)$ and the eigenphase sums were also investigated in order to study the resonant behavior of the partial cross sections for some particular symmetries of $\mathrm{CS}_{2}$.

In the next sections we present the theoretical formulation of our method, our computational procedures and our results and discussions. We end this work with a brief summary. 


\section{Theoretical Formulation}

Here we will give a brief description of the SMC $[8,9]$ and SMCPP [6] methods. The SMC method is a multichannel extension of the Schwinger variational principle. Actually, it is a variational approximation for the scattering amplitude, where the scattering wave function is expanded in a basis of $(\mathrm{N}+1)$-particle Slater determinants

$$
\left|\Psi_{\vec{k}}\right\rangle=\sum_{m} a_{m}^{ \pm}(\vec{k})\left|\chi_{m}\right\rangle
$$

and the coefficients $a_{m}^{ \pm}(\vec{k})$ of this expansion are variationally determined. The resulting expression for the scattering amplitude in the body frame is

$\left[f_{\vec{k}_{i}, \vec{k}_{f}}\right]=-\frac{1}{2 \pi} \sum_{m, n}\left\langle S_{\vec{k}_{f}}|V| \chi_{m}\right\rangle\left(d^{-1}\right)_{m n}\left\langle\chi_{n}|V| S_{\vec{k}_{i}}\right\rangle$,

where

$$
d_{m n}=\left\langle\chi_{m}\left|A^{(+)}\right| \chi_{n}\right\rangle
$$

and

$$
A^{(+)}=\frac{\hat{H}}{N+1}-\frac{(\hat{H} P+P \hat{H})}{2}+\frac{(V P+P V)}{2}-V G_{P}^{(+)} V .
$$

In the above equations $\left|S_{\vec{k}}\right\rangle$, solution of the unperturbed Hamiltonian $H_{0}$, is the product of a target state and a plane wave, $V$ is the interaction potential between the incident electron and the target, $\left|\chi_{m}\right\rangle$ is an $(\mathrm{N}+1)$ electron Slater determinant used in the expansion of the trial scattering wave function, $\hat{H}=E-H$ is the total energy of the collision minus the full Hamiltonian of the system, with $H=H_{0}+V . P$ is a projection operator onto the open-channel space defined in terms of target eigenfunctions $\left|\Phi_{l}\right\rangle$ :

$$
P=\sum_{l}^{\text {open }}\left|\Phi_{l}\right\rangle\left\langle\Phi_{l}\right|
$$

and $G_{P}^{(+)}$is the free-particle Green's function projected on the $P$-space.

In this paper, we study elastic scattering at the static-exchange approximation, and the operator $P$ is composed only by the ground state of the target $\left|\Phi_{1}\right\rangle$

$$
P=\left|\Phi_{1}\right\rangle\left\langle\Phi_{1}\right|
$$

and the configuration space $\left|\chi_{m}\right\rangle$ is

$$
\left\{\left|\chi_{m}\right\rangle\right\}=\left\{\mathcal{A}\left|\Phi_{1}\right\rangle\left|\varphi_{m}\right\rangle\right\}
$$

where $\left|\varphi_{i}\right\rangle$ is a 1-particle function represented by one molecular orbital.

With the choice of Cartesian Gaussian functions to represent the molecular and scattering orbitals, all the matrix elements arising in Eq. (2) can be computed analytically, except those from $\left\langle\chi_{m}\left|V G_{P}^{(+)} V\right| \chi_{n}\right\rangle$ (VGV), that are evaluated by numerical quadrature [9].

The numerical calculation of the matrix elements from VGV represents the most expensive step in the
SMC code and demands almost the entire computational time of the scattering calculation. These matrix elements are reduced to a sum of primitive two-electron integrals involving a plane wave and three Cartesian Gaussian functions

$$
\langle\alpha \beta|V| \gamma \vec{k}\rangle=\iint d \vec{r}_{1} d \vec{r}_{2} \alpha\left(\vec{r}_{1}\right) \beta\left(\vec{r}_{1}\right) \frac{1}{r_{12}} \gamma\left(\vec{r}_{2}\right) e^{i \vec{k} \cdot \vec{r}_{2}},
$$

and must be evaluated for all possible combinations of $\alpha, \beta$ and $\gamma$ and for several directions and moduli of $\vec{k}$. We must also evaluate one-electron integrals of the type

$$
\left\langle\alpha\left|V^{P P}\right| \vec{k}\right\rangle=\int d \vec{r} \alpha(\vec{r}) V^{P P} e^{i \vec{k} \cdot \vec{r}} .
$$

In the above equation, $V^{P P}$ is the nonlocal pseudopotential operator given by:

$$
\hat{V}^{P P}(r)=\hat{V}_{\text {core }}(r)+\hat{V}_{\text {ion }}(r),
$$

with

$$
\hat{V}_{\text {core }}(r)=-\frac{Z_{v}}{r}\left[\sum_{i=1}^{2} c_{i}^{\text {core }} \operatorname{erf}\left[\left(\alpha_{i}^{\text {core }}\right)^{1 / 2} r\right]\right],
$$

and

$\hat{V}_{i o n}(r)=\sum_{n=0}^{1} \sum_{j=1}^{3} \sum_{l=0}^{2} A_{n j l} r^{2 n} e^{-\alpha_{j l} r^{2}} \sum_{m=-l}^{+l}|l m\rangle\langle l m|$,

where $Z_{v}$ is the valence charge of the atom and in this application it is equal to 4 for $\mathrm{C}$, and 6 for $\mathrm{S}$. The coefficients $c_{i}^{\text {core }}, A_{n j l}$, and the decay constants $\alpha_{i}^{\text {core }}$ and $\alpha_{j l}$ are tabulated in Ref. [10]. 
Even for small molecules composed by light atoms, a large number of the two-electron integrals must be evaluated. This limits the size of molecules in scattering calculations. In the SMCPP method we need shorter basis set to describe the target and scattering and consequently the number of two electron integrals is smaller than in the all-electron case. The one-electron integrals of Eq. (9) are more complex than those involving the nuclei, but they can be calculated analytically and their number is also reduced due to the smaller basis set. The reduction in the number of these integrals allows the study of molecules that are larger and heavier than those reachable by all-electron techniques.

\section{Computational Procedures}

The ground state of the molecule, ${ }^{1} \Sigma_{g}$, is described by a single-configuration wave function $\left|\Phi_{1}\right\rangle$ (HartreeFock level) at the experimental geometry with $r(\mathrm{C}$ $\mathrm{S})=1.5526 \AA$ [11]. The $1 s$ core electrons of carbon and the $1 s, 2 s$, and $2 p$ core electrons of sulfur were replaced by the pseudopotentials of Ref. [10]. The basis functions used in the description of the target ground state $\left|\Phi_{1}\right\rangle$ and in the description of the scattering orbitals $\left|\varphi_{i}\right\rangle$ used in Eq. ( 7) are given in Table 1, and were obtained as described in Ref. [12]. We have not included in our calculations the symmetrical combination of $d$ functions $\left(\left[\left(x^{2}+y^{2}+z^{2}\right) \exp \left(-\alpha r^{2}\right)\right]\right)$, which is in fact a s-type function, in order to avoid the linear dependency in the basis set that could be responsible for spurious structures in the cross sections [13].

Table 1. Cartesian Gaussian functions for carbon and sulfur

\begin{tabular}{crc} 
& $\mathrm{C}$ & $\mathrm{S}$ \\
Type & Exponent & Exponent \\
\hline $\mathrm{s}$ & 12.494080 & 7.382257 \\
$\mathrm{~s}$ & 2.470291 & 2.063167 \\
$\mathrm{~s}$ & 0.614027 & 0.878009 \\
$\mathrm{~s}$ & 0.184029 & 0.245161 \\
$\mathrm{~s}$ & 0.036799 & 0.061630 \\
$\mathrm{~s}$ & 0.013682 & 0.015560 \\
\hline $\mathrm{p}$ & 5.228869 & 7.203417 \\
$\mathrm{p}$ & 1.592058 & 3.134723 \\
$\mathrm{p}$ & 0.568612 & 0.529380 \\
$\mathrm{p}$ & 0.210326 & 0.154155 \\
$\mathrm{p}$ & 0.072250 & 0.035523 \\
\hline $\mathrm{d}$ & 0.831084 & 1.689035 \\
$\mathrm{~d}$ & 0.229204 & 0.476317 \\
$\mathrm{~d}$ & 0.075095 & 0.151558 \\
${ }^{a}$ Cartesian Gaussian functions are defined by \\
$\phi_{l m n}=N_{l m n}\left(x-a_{x}\right)^{l}\left(y-a_{y}\right)^{m}\left(z-a_{z}\right)^{n} \exp \left(-\alpha|\vec{r}-\vec{a}|^{2}\right)$
\end{tabular}

$\phi_{l m n}=N_{l m n}\left(x-a_{x}\right)^{l}\left(y-a_{y}\right)^{m}\left(z-a_{z}\right)^{n} \exp \left(-\alpha|\vec{r}-\vec{a}|^{2}\right)$

\section{Results and Discussion}

Fig. 1 shows the comparison between our integral cross section, the calculated cross section of Lynch et al. [1] and Lee et al. [5], and the measured total cross section of Szmytkowski [2]. We also show the data at $5 \mathrm{eV}$ of Sohn et al. [3]. There is very good agreement between our results and the results of Lynch et al. for energies above $15 \mathrm{eV}$. Both calculations agree with the result of Sohn et al. at $5 \mathrm{eV}$, and also follow the shape of the total experimental cross section data of Szmytkowski. For energies between $5 \mathrm{eV}$ and $15 \mathrm{eV}$, the agreement between both these theoretical results is qualitative. The cross section of Lee $e t$ al. is above our cross sections and the cross section of Lynch et al. for the entire energy range considered. The structures in our integral cross section are related to structures in the partial cross sections for particular symmetries, as discussed below.

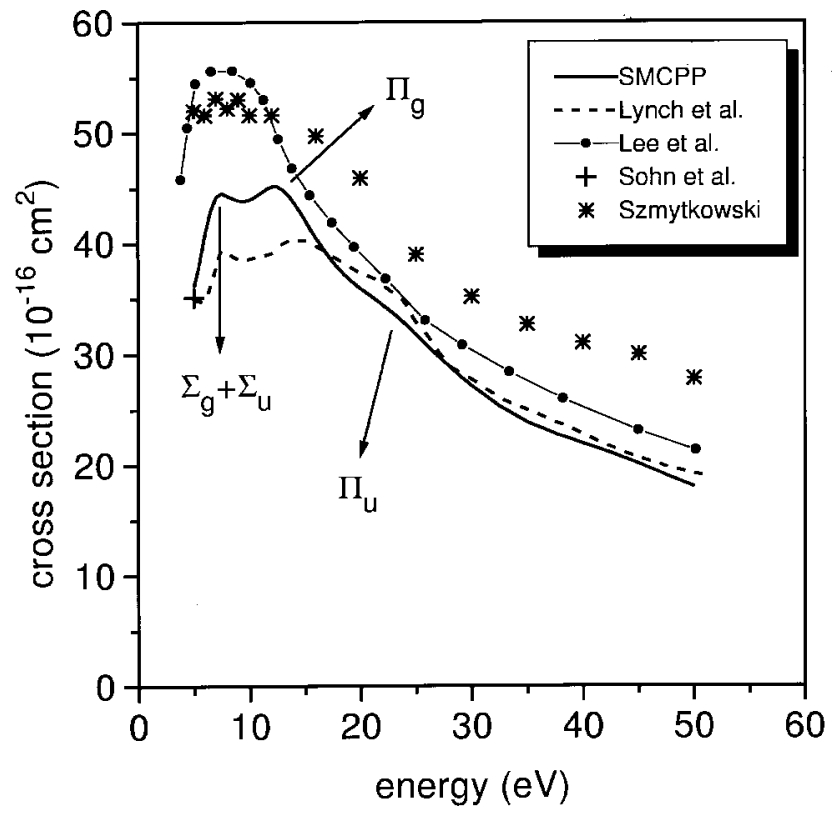

Figure 1. Integral cross section for $\mathrm{CS}_{2}$. Solid line, our pseudopotential results; dashed line, theoretical results of Ref. [1]; thin solid line with circles, theoretical results of Ref. [5]; plus sign, experimental elastic datum of Ref. [3]; stars, experimental total cross section of Ref. [2]. The arrows indicate the structures positions and the symmetries they appear are also shown.

In Fig. 2 we show the symmetry decomposition of our integral cross section according to the irreducible representations of the $\mathrm{D}_{\infty h}$ point group. Although not shown, our partial cross sections are in good agreement with those of Ref. [1]. It is clear in this figure that the structures in our integral cross section are related to the structures that appear in the symmetries $\Sigma_{g}, \Sigma_{u}$, $\Pi_{g}$, and $\Pi_{u}$, at $7 \mathrm{eV}, 6.5 \mathrm{eV}, 13.5 \mathrm{eV}$, and $23 \mathrm{eV}$, respectively, as indicated by the arrows in Fig. 1. The $\Pi_{u}$ structure produces a very smooth undulation in our 
integral cross section due to the significant background from other symmetries at this energy.

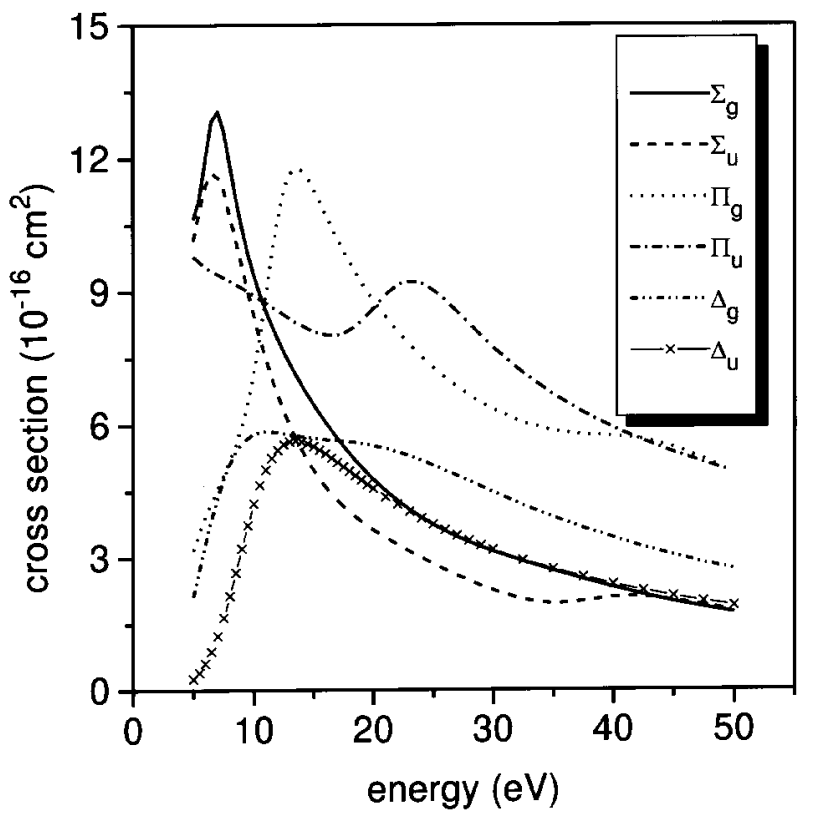

Figure 2. Symmetry decomposition of the integral cross section.

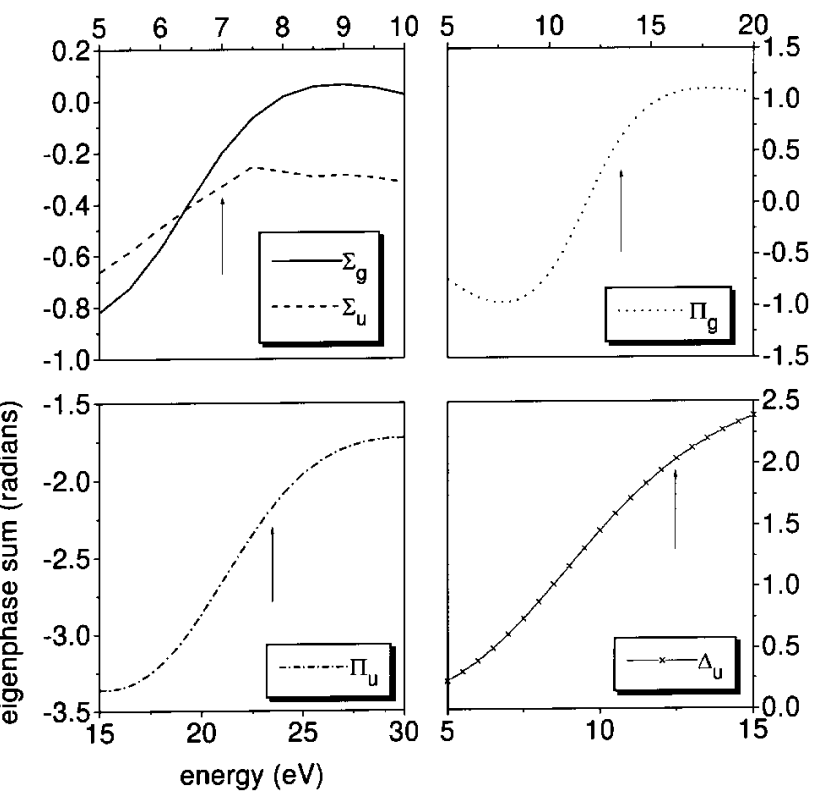

Figure 3. Eigenphase sum for the $\Sigma_{g}, \Sigma_{u}, \Pi_{g}, \Pi_{u}$ and $\Delta_{u}$ symmetries. The arrows indicate the peaks position of the partial cross sections of Fig. 2. The peaks for the $\Sigma_{g}$ and $\Sigma_{u}$ symmetries are located at the same energy.

In order to study possible resonant behavior in our partial cross sections we have also calculated the eigenphase sum for each one of the symmetries. Our results are shown in Fig. 3 for possible resonant symmetries (those with bumps in Fig. 2). For each symmetry, we mark with an arrow the energy at the peak and it is always close to the maximum slope of the eigenphase. Therefore, this figure indicates that the structures referred to in the previous paragraph for the symmetries $\Sigma_{g}, \Pi_{g}$, and $\Pi_{u}$ may be, in fact, broad shape resonances. In the case of $\Delta_{u}$, the structure located at $12.5 \mathrm{eV}$, is very broad and cannot be seen in the integral cross section due to its very low magnitude. Lynch et al. also reported the $\Sigma_{g}$ resonance, placed at $7.9 \mathrm{eV}$. The $\Pi_{g}, \Pi_{u}$, and $\Delta_{u}$ resonances are being identified in this work for the first time. In the case of $\Sigma_{u}$, there is a spurious and very sharp resonance around $8.0 \mathrm{eV}$ which is resposible for the slope discontinuity of the eigenphase sum in Fig. 3. We are not interpreting that discontinuity as a real resonance because the phase change is too small.

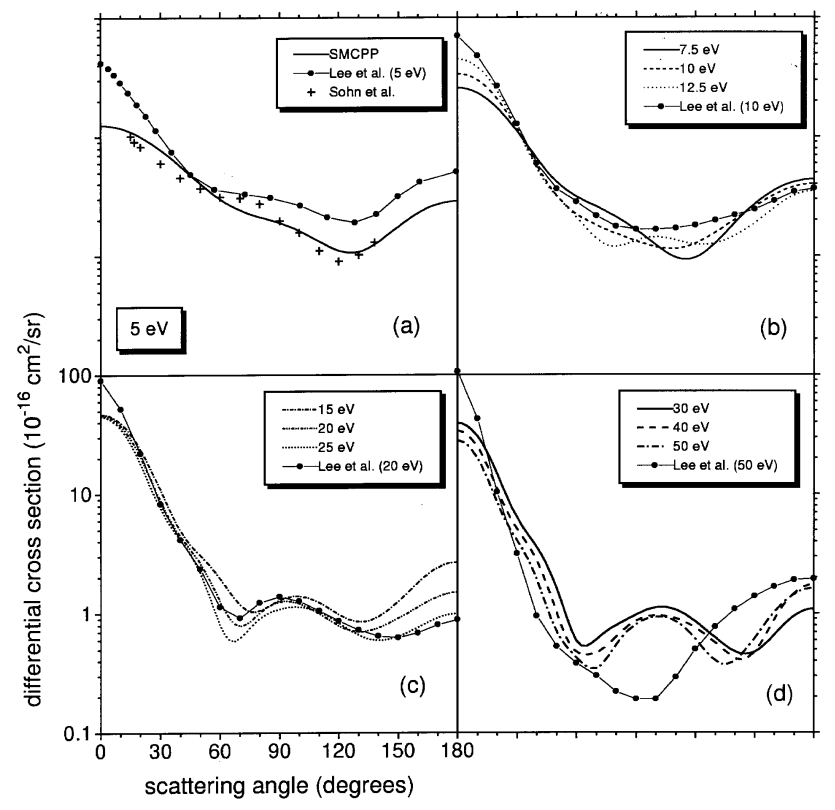

Figure 4. Differential cross sections for $\mathrm{CS}_{2}$. Lines, our pseudopotential results; thin solid line with circles, theoretical results of Ref. [5]; plus signs, experimental elastic data of Ref. [3].

In Fig. 4a we compare our differential cross section (DCS) with the measured DCS of Sohn et al. and calculated DCS by Lee et al. at $5 \mathrm{eV}$. There is very good agreement between our results and the results of Sohn et al.. Although the DCS of Lee et al. presents the same shape than ours, it is greater in magnitude than ours at all scattering angles. In Figs. $4 \mathrm{~b}, 4 \mathrm{c}$, and $4 \mathrm{~d}$ we show our calculated DCS at 7.5, 10, 12.5, 15, 20, 25, 30, 40 and $50 \mathrm{eV}$ and calculated DCS of Lee et al. at 10,20 , and $50 \mathrm{eV}$. At 10 and $20 \mathrm{eV}$ we found good agreement in shape with the DCS of Lee et al.. For energies above $10 \mathrm{eV}$, our the DCS present two minima, indicating a $d$-wave behavior. 
Finally, Fig. 5 shows our momentum transfer cross section. There is no available results for comparison. In Table 2 we present our results for differential, integral, and momentum transfer cross sections at selected energies.

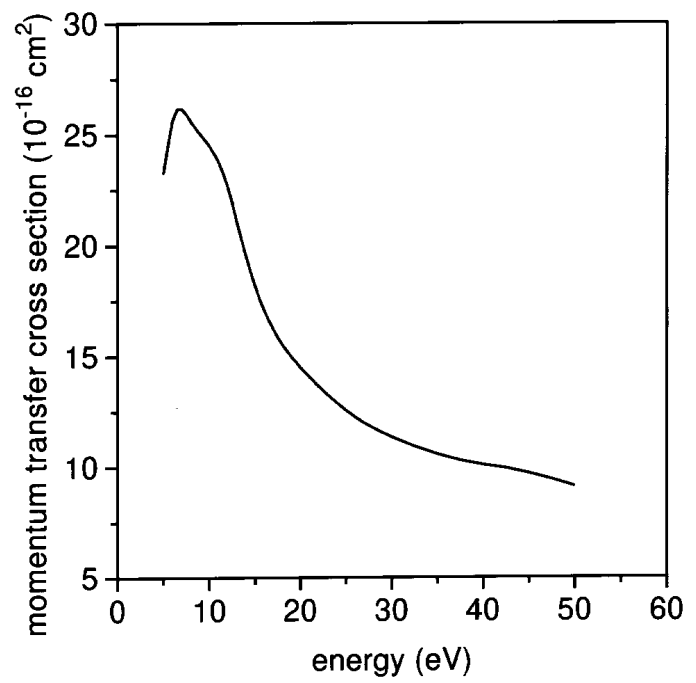

Figure 5. Momentum transfer cross section.

\section{Summary}

We presented results for elastic scattering of low-energy electrons by $\mathrm{CS}_{2}$ molecules. Our results agree very well with the available theoretical and experimental results. Through the symmetry decomposition of the integral cross section and eigenphase sum analysis we tentatively assign the cross section peaks to broad shape resonances for the $\Sigma_{g}, \Pi_{g}, \Pi_{u}$, and $\Delta_{u}$ symmetries, at $7 \mathrm{eV}, 13.5 \mathrm{eV}, 23 \mathrm{eV}$, and $12.5 \mathrm{eV}$ respectively. In this work, we have identified these resonances for the first time, except for the $\Sigma_{g}$ one, reported by Lynch et al. [1] at $7.9 \mathrm{eV}$.

\section{Acknowledgments}

M.H.F.B., L.G.F. and M.A.P.L. acknowledge support from CNPq. A. P. P. N. acknowledges support from FAPESP. M.H.F.B. also acknowledges partial support from FUNPAR. Our calculations were performed at CENAPAD-SP, at CENAPAD-NE and at CCEUFPR. The authors acknowledge Mr. Márcio T. do N. Varella for his suggestions concerning this manuscript and Mr. Marlus Koehler for digitizing the results of Lynch et al.

Table 2. Tabulated cross sections for $\mathrm{CS}_{2}$ at selected energies. The cross sections are in $10^{-16} \mathrm{~cm}^{2}$ and the scattering angles are in degrees.

\begin{tabular}{rrrrrrrrrrr}
\hline angle & $5 \mathrm{eV}$ & $7.5 \mathrm{eV}$ & $10 \mathrm{eV}$ & $12.5 \mathrm{eV}$ & $15 \mathrm{eV}$ & $20 \mathrm{eV}$ & $25 \mathrm{eV}$ & $30 \mathrm{eV}$ & $40 \mathrm{eV}$ & $50 \mathrm{eV}$ \\
\hline 0.0 & 12.58 & 25.78 & 34.01 & 44.99 & 47.26 & 46.42 & 45.22 & 39.55 & 34.03 & 28.17 \\
10.0 & 11.88 & 23.31 & 30.02 & 38.52 & 39.81 & 37.75 & 35.62 & 30.49 & 24.90 & 20.00 \\
20.0 & 10.08 & 17.38 & 20.76 & 24.28 & 23.93 & 20.71 & 18.04 & 14.75 & 10.95 & 8.51 \\
30.0 & 7.82 & 11.05 & 11.57 & 11.80 & 10.92 & 8.91 & 7.60 & 6.34 & 5.07 & 4.07 \\
40.0 & 5.70 & 6.53 & 5.75 & 5.40 & 4.99 & 4.48 & 4.19 & 3.57 & 2.77 & 1.91 \\
50.0 & 4.07 & 4.16 & 3.17 & 3.14 & 3.08 & 2.65 & 2.20 & 1.62 & 1.02 & 0.71 \\
60.0 & 3.01 & 3.12 & 2.22 & 2.10 & 1.98 & 1.28 & 0.82 & 0.59 & 0.47 & 0.43 \\
70.0 & 2.43 & 2.58 & 1.80 & 1.39 & 1.19 & 0.79 & 0.63 & 0.60 & 0.47 & 0.34 \\
80.0 & 2.13 & 2.11 & 1.52 & 1.19 & 1.06 & 1.02 & 0.91 & 0.79 & 0.63 & 0.55 \\
90.0 & 1.90 & 1.64 & 1.32 & 1.33 & 1.30 & 1.26 & 1.09 & 0.97 & 0.86 & 0.81 \\
100.0 & 1.63 & 1.22 & 1.18 & 1.43 & 1.40 & 1.21 & 1.13 & 1.12 & 0.96 & 0.93 \\
110.0 & 1.33 & 0.97 & 1.14 & 1.34 & 1.24 & 1.00 & 1.04 & 1.07 & 0.90 & 0.85 \\
120.0 & 1.11 & 0.96 & 1.26 & 1.24 & 0.99 & 0.79 & 0.84 & 0.85 & 0.76 & 0.61 \\
130.0 & 1.09 & 1.27 & 1.56 & 1.27 & 0.86 & 0.71 & 0.66 & 0.62 & 0.57 & 0.40 \\
140.0 & 1.31 & 1.89 & 2.01 & 1.47 & 0.94 & 0.76 & 0.60 & 0.48 & 0.42 & 0.40 \\
150.0 & 1.76 & 2.70 & 2.59 & 1.88 & 1.26 & 0.91 & 0.64 & 0.48 & 0.47 & 0.62 \\
160.0 & 2.28 & 3.54 & 3.24 & 2.52 & 1.81 & 1.13 & 0.77 & 0.66 & 0.88 & 1.02 \\
170.0 & 2.71 & 4.16 & 3.80 & 3.20 & 2.41 & 1.38 & 0.92 & 0.95 & 1.47 & 1.44 \\
180.0 & 2.87 & 4.39 & 4.03 & 3.51 & 2.69 & 1.50 & 1.00 & 1.09 & 1.75 & 1.63 \\
\hline ics & 36.12 & 44.53 & 43.98 & 45.12 & 42.08 & 35.92 & 31.75 & 27.13 & 21.92 & 17.98 \\
\hline mtcs & 23.25 & 25.92 & 24.50 & 21.93 & 18.18 & 14.49 & 12.56 & 11.35 & 10.10 & 9.13 \\
\hline
\end{tabular}




\section{References}

[1] M. G. Lynch, and D. Dill, J. Chem. Phys. 71, 4249 (1979).

[2] C. Szmytkowski, J. Phys. B: At. Mol. Phys. 20, 6613 (1987).

[3] W. Sohn, K-H. Kochem, K. M. Scheuerlein, K. Jung, and H. Ehrhadt, J. Phys. B: At. Mol. Phys. 20, 3217 (1987).

[4] D. Raj, and S. Tomar, J. Phys. B: At. Mol. Opt. Phys. 30, 1989 (1997).

[5] M. T. Lee, S. E. Michelin, T. Kroin, and E. Veitenheimer, J. Phys. B: At. Mol. Opt. Phys. 32, 3043 (1999).

[6] M. H. F. Bettega , L. G. Ferreira, and M. A. P. Lima, Phys. Rev. A 47, 1111 (1993).

[7] M. H. F. Bettega, M. A. P. Lima, and L. G. Ferreira, J. Phys. B: At. Mol. Opt. Phys. 31, 2091 (1998); M. H. F. Bettega, L. G. Ferreira, and M. A. P. Lima, Phys. Rev. A 57, 4987 (1998); S. M. S. da Costa, and M. H. F. Bettega, Eur. Phys. J. D 3, 67 (1998); M. H. F. Bettega, M. T. do N. Varella, L. G. Ferreira, and M. A. P. Lima,
J. Phys. B: At. Mol. Opt. Phys 31, 4419 (1998); A. P. P. Natalense, L. G. Ferreira, and M. A. P. Lima, Phys. Rev. Lett. 81, 3832 (1998); A. P. P. Natalense, M. H. F. Bettega, L. G. Ferreira, and M. A. P. Lima, Phys. Rev. A 59, 879 (1999); M. T. do N. Varella, M. H. F. Bettega, A. J. R. da Silva, and M. A. P. Lima, J. Chem. Phys. 110, 2452 (1999).

[8] K. Takatsuka, and V. McKoy, Phys. Rev. A 24, 2473 (1981); ibid. 30, 1734 (1984).

[9] M. A. P. Lima, L. M. Brescansin, A. J. R. da Silva, C. Winstead, and V. McKoy, Phys. Rev. A 41, 327 (1990).

[10] G. Bachelet, D. R. Hamann, and M. Schlüter, Phys. Rev. B 26, 4199 (1982).

[11] CRC Handbook of Chemistry and Physics, D. R. Lide Editor in Chief, $73^{\text {rd }}$ ed.

[12] M. H. F. Bettega, A. P. P. Natalense, M. A. P. Lima, and L. G. Ferreira, Int. J. Quantum Chem. 60, 821 (1996).

[13] M. T. do N. Varella, A. P. P. Natalense, M. H. F. Bettega, and M. A. P. Lima, Phys. Rev. A 60, 3684 (1999). 